\title{
LA MUJER Y EL SISTEMA EDUCATIVO EN COLOMBIA
}

Elssy Bonilla de Ramos*

\section{Introducción}

El análisis de cualquier aspecto relacionado con el sistema educativo, debe partir de la ubicación de éste dentro de las condiciones históricas de la sociedad que se considere. Esto por cuanto el sistema educativo se estructura como un medio para responder a la necesidad de formar una fuerza de trabajo entrenada y disciplinada, según el grado de desarrollo de las fuerzas productivas en una formación social históricamente determinada $^{10}$. Es decir, la educación se estructura para cumplir papeles determinados según condiciones históricas también determinadas.

Paralelamente con esta argumentación, es necesario considerar que las ventajas y limitaciones del sistema educativo no pueden considerarse aisladamente como aspectos inherentes al sistema mismo, sino que, por el contrario, responden a situaciones concretas de la sociedad donde funciona dicho sistema. Además, desubicarlas de este contexto, equivale a limitar — cuando no a impedir - las posibilidades de comprender el por qué de dichas ventajas y de dichas limitaciones.

El sistema educativo de una sociedad dividida en clases, con intereses contradictorios, no puede ser ajeno a las desigualdades intrínsecas que surgen de las relaciones entre estas clases. Es precisamente dentro de este contexto en donde se ubica la desigual participación de la mujer en la educación y es en el mismo, como lo veremos más adelante, donde se deben enfocar las políticas de acción para la mujer que traten de redefinir su participación, no solo a nivel del sistema educativo, sino de la sociedad total.

Estas consideraciones han llevado a que algunos estudios, en su búsqueda de una comprensión de por qué la mujer es discriminada en el sistema educativo, superen aquellos esfuerzos académicos orientados básicamente a declaraciones de denuncia acerca de la opresión de la mujer por parte del hombre y en donde, aun cuando se describan las formas en las cuales se efectúa esta discriminación, no llegan a una comprensión cabal ni a explicar adecuadamente las razones de este hecho.

\section{La mujer en el sistema educativo}

El estudio de la participación de la mujer en el desarrollo económico nacional, ha surgido generalmente de los supuestos de que el desarrollo lleva necesariamente a una

\footnotetext{
Socióloga Universidad Nacional, P.H.D. Comunicaciones y Desarrollo; Investigadora del CEDE Universidad de los Andes.

${ }^{10}$ Se considera en efecto, que en sociedades precapitalistas la formación de la fuerza de trabajo se daba en el seno de la familia, la cual constituía también un núcleo básico en el proceso de producción. Con el desarrollo del sistema capitalista, el trabajo se desplaza a la fábrica y la educación a la escuela. Ver: Samuel Bowles, Unequal Education and the Reproduction of the Hierarchical Division of Labor en Richard Edwards y otros. The Capitalist System, New Jersey, Prentice Hall, Inc., 1972. También, Elssy Bonilla de Ramos, Class Struggle and the Transmission of Ideology. An Analysis of the Colombian' School System. Ph. D. Theals. Madison. University of Wisconsin. 1976.
} 
disminución de las desigualdades sociales por un lado, y por otro, de que éste requiere de la capacitación de toda la población. Partiendo de esta doble tesis ha llegado a plantearse que la limitada participación de la mujer en el sistema educativo (su falta de capacitación), es una de las razones fundamentales por las cuales ella ha alcanzado una cuota de participación tan limitada en los procesos de desarrollo nacional. Este planteamiento pierde de vista el hecho de que no todos los sectores de la población femenina han sido excluidos de una participación en el desarrollo, ni del sistema escolar, y que por lo tanto la discriminación no se ha ejercido de manera homogénea, ni puede superarse con soluciones homogéneas para toda la población femenina. Esto, por cuanto si bien es cierto que existe una discriminación contra la mujer, que sería necio negar, esta discriminación se ejerce de manera diferente para mujeres de diversas posiciones socioeconómicas y afecta no solamente a la mujer, sino en grado significativo también al hombre.

Es decir, que si bien no puede negarse una discriminación por sexo, el sexo no es la base de las discriminaciones sociales, sino fundamentalmente la posición del individuo y en nuestro caso concreto de la mujer, dentro de una clase social. Aquí se enmarca uno de los aspectos fundamentales de la influencia de la familia en la educación: la posición de esa familia dentro de una red de relaciones de clase históricamente determinadas.

Según este marco de análisis planteado, se ubicará la posición de la mujer en el sistema educativo teniendo en cuenta la participación femenina en los distintos niveles educativos, donde por ley debe tener una participación similar a la del hombre.

El análisis de los aspectos legales de la educación no pueden ubicarse en abstracto, sino en las condiciones históricas concretas en las que fueron sancionadas. Como Molano ${ }^{11}$ ha señalado, una visión histórica de la educación no puede reducirse a un catálogo de eventos supuestos, a una colección de leyes y decretos o a una exaltación de educadores. El problema va más allá de eso. Es necesario robar el secreto de la lógica interna que explica estos eventos en su verdadero significado, las fases de la legislación y las razones que, desconocidas por los grandes hombres, terminaron imponiendose sobre ellos.

Sin embargo, como señala Rama ${ }^{12}$, en su análisis del origen social de la población universitaria en Colombia, el sistema educativo vigente adjudica de hecho a cada grupo social un nivel específico de conocimientos, dificulta el logro de otros niveles más altos o distintos que los que el sistema presupone para cada grupo y establece implícitamente como criterio de promoción la pertenencia a ciertos grupos sociales.

El mismo autor señala cómo la participación de la mujer en la educación universitaria es altamente significativa para aquellas que provienen de grupos familiares cuyos abuelos, padres y hermanos han obtenido o están en proceso de obtener un título universitario. En efecto, "el sexo femenino muestra una tasa mayor de pertenencia a grupos familiares universitarios, ya que en un $71.5 \%$ tienen hermanos realizando o que realizaron estudios universitarios y en un $78.1 \%$ tienen parientes graduados en las universidades".

\footnotetext{
${ }^{11}$ Molano, Alfredo. Economía y Educación en 1850. Bogotá 1974. OFISEL.

${ }^{12}$ Rama. Germán. "Origen social de la población universitaria". En Gonzalo Cataño. Educación y Sociedad en Colombia. Bogotá, 1973. Universidad Pedagógica, p. 199.
} 
Las condiciones de la mujer frente al sistema educativo no son las mismas para todas las mujeres, sino que dependen de su origen de clase, y la discriminación del sistema educativo no es fundamentalmente una discriminación por sexo, sino esencialmente una de clase.

Si se analizan por ejemplo los datos de analfabetismo (Tabla 1), puede observarse que si bien las tasas de analfabetismo femenino son más altas q6e las masculinas, es imposible sin embargo afirmar que el hombre no ha sido también discriminado del sistema educativo. Más aún, estos datos muestran que la naturaleza de la discriminación es mucho más significativa cuando se comparan las tasas urbanas y rurales que cuando se comparan las tasas por sexos.

\section{TABLA I}

TASAS DE ANALFABETISMO EN COLOMBIA

\begin{tabular}{|l|c|c|r|r|}
\hline \multirow{2}{*}{ Año } & \multicolumn{2}{|c|}{ URBANA } & \multicolumn{2}{c|}{ RURAL } \\
\cline { 2 - 5 } & Hombre & Mujer & Hombre & Mujer \\
\hline 1951 & 16.6 & 24.5 & 46.1 & 53.6 \\
\hline 1964 & 11.9 & 17.4 & 38.4 & 44.4 \\
\hline
\end{tabular}

Fuente: $\quad$ Censos de población para 1951 y 1964.

\section{A. Nivel de primaria}

Analizando la participación de la mujer en la educación primaria, es posible constatar que en los 10 años correspondientes entre 1964 y 1974 ésta ha tenido una participación similar a la del hombre y que tanto hombres como mujeres han sido discriminados de manera similar en el sistema escolar.

Como puede observarse en la Tabla 2, la educación primaria pasó de un total de $56.7 \%$ de la población en edad escolar que cubría en 1964, a 68.6\% de la población en 1974 . Estos datos muestran un porcentaje bastante similar entre la población masculina y femenina entre los 7 y los 14 años. Sin embargo, y a pesar de la expansión observada en 1974 , más del $30 \%$ de la población que debería participar en la educación primaria, queda fuera del alcance de ésta. 


\title{
TABLA II \\ EVOLUCION DE LA MATRICULA EN \\ LA EDUCACION PRIMARIA POR SEXO, 1964-1974
}

\begin{abstract}
Total Población entre 7-14 AñosMatriculados entre 7-14 Años
Años Total Hombres Mujeres Total Hombres Mujeres

$7-14 \quad 7.14 \quad 7-14$
\end{abstract}

\begin{tabular}{|c|c|c|c|c|c|c|}
\hline & \multicolumn{3}{|c|}{ Total población entre 7 - 14 años } & \multicolumn{3}{c|}{ Matriculados entre 7-14 años } \\
\cline { 2 - 7 } Años & $\begin{array}{c}\text { Total } \\
\mathbf{7 - 1 4}\end{array}$ & $\begin{array}{c}\text { Hombres } \\
\mathbf{7 - 1 4}\end{array}$ & $\begin{array}{c}\text { Mujeres } \\
\mathbf{7 - 1 4}\end{array}$ & $\begin{array}{c}\text { Total } \\
\text { \% }\end{array}$ & $\begin{array}{c}\text { Hombres } \\
\text { \% }\end{array}$ & $\begin{array}{c}\text { Mujeres } \\
\text { \% }\end{array}$ \\
\hline 1964 & 3.907 .315 & 1.973 .194 & 1.934 .121 & 56.7 & 56.2 & 57.2 \\
\hline 1965 & 4.037 .437 & 2.038 .906 & 1.998 .531 & 56.3 & 55.3 & 57.4 \\
\hline 1966 & 4.173 .813 & 2.111 .950 & 2.061 .863 & 57.5 & 56.6 & 58.5 \\
\hline 1967 & 4.316 .744 & 2.184 .272 & 2.132 .472 & 59.9 & 59.1 & 60.7 \\
\hline 1968 & 4.466 .546 & 2.260 .072 & 2.206 .474 & 61.2 & 60.5 & 62.1 \\
\hline 1969 & 4.623 .547 & 2.339 .515 & 2.284 .032 & 67.2 & 66.4 & 66.8 \\
\hline 1970 & 4.788 .098 & 2.408 .413 & 2.379 .685 & 88.6 & 68.2 & 69.1 \\
\hline 1971 & 4.960 .551 & 2.945 .157 & 2.465 .394 & 70.8 & 71.4 & 70.3 \\
\hline 1972 & 5.141 .284 & 2.586 .066 & 2.555 .218 & 69.4 & 69.2 & 69.7 \\
\hline 1973 & 5.330 .690 & 2.681 .337 & 2.649 .353 & 70.0 & 69.9 & 70.1 \\
\hline 1974 & 5.529 .176 & 2.781 .175 & 2.748 .001 & 68.6 & 68.6 & 68.5 \\
\hline
\end{tabular}

Fuentes: DANE. Anuario General de Estadísticas Culturales 1963 a 1967; Censo Educativo 1968 Oficial Urbana y Rural; Proyecciones $1969=1971$ y $1972=1974$ Secretaría de Educación Urbana y Rural 1969 a 1974; Boletín Mensual de Estadística No. 288 de Julio de 1975; Educación Primaria en Colombia 1967 = 1974, Bogotá, 1975.

A nivel de la primaría, sin embargo, y a pesar de la apertura atrás señalada, la discriminación educativa se hace mucho más evidente cuando se visualizan las posibilidades de permanecer en el sistema y cuando estas posibilidades se contrastan entre lo urbano y lo rural.

En relación con el primer aspecto, son conocidos los datos de permanencia en el sistema. En efecto, de cada 100 niños en edad escolar $23 \%$ nunca fueron a la escuela, $77 \%$ se registraron en primero, $50.5 \%$ en segundo, $35.7 \%$ en tercero, $26.3 \%$ encuarto y $21.6 \%$ en quinto año ${ }^{13}$.

Estos datos se tornan especialmente dicientes sobre la naturaleza de la discriminación del sistema educativo colombiano cuando se analizan por áreas rural y urbana. Con base en datos de $1964^{14}$, se quedó fuera del sistema escolar el $27 \%$ de la población mayor de 15 años. Ello significaba el $15 \%$ de la población urbana y el $41.3 \%$ de la población rural.

Esta situación de discriminación se hace más aguda cuando se establece que si bien $39.2 \%$ de los niños en las áreas urbanas terminan el ciclo primario, en las áreas rurales solo lo logra el $9.7 \%$. Dada la homogénea participación por sexos antes señalada, nos encontramos que tanto los hombres como las mujeres son discriminados del sistema

13 Departamento Nacional de Estadística (1970:3). Esta misma fuente considera que $64.3 \%$ de todos los niños entre los 7-14 años que tuvieron 2 o menos años de escuela, se consideran analfabetas funcionales.

${ }_{14}$ Datos presentados por Rama, Op. cit. 
educativo a nivel primario y que lo son aún más los hombres y las mujeres de las áreas rurales que los hombres y las mujeres de las áreas urbanas.

\section{B. Nivel de secundaria}

El análisis de la participación de la mujer en la educación media se realizará teniendo en cuenta lo siguiente:

a) Esta ofrece dos tipos de orientación básica, una hacia la capacitación para el desempeño de determinadas ocupaciones (comercial, normalista, industrial, etc.) y otra transicional hacia la educación superior.

b) Como lo ha señalado el DANE$^{15}$ "tras los objetivos explícitos, la educación media cumple con funciones que se desprenden de la estructuración de la sociedad. Es en este sentido, un instrumento de reproducción de las condiciones económicas, sociales e ideológicas, es un mecanismo de diferenciación social y de transmisión de los contenidos de la cultura predominante".

El primer aspecto señalado plantea la existencia de una clara relación entre la naturaleza de la participación en la educación media y la ubicación futura del individuo en la división social del trabajo. Esto implica, en relación con la mujer, que la educación a nivel medio se constituye para un alto porcentaje de ellas, en su máxima aspiración. Pero, teniendo en cuenta el segundo aspecto señalado, no toda la población femenina que alcanza los niveles medios está en igualdad de condiciones para decidir por una educación media terminal o por una transitoria que le permita la formación universitaria.

Por un lado tenemos que según la ubicación de clase, la mujer cuenta con diferentes posibilidades para participar en el sistema educativo y, concomitantemente, de orientarse hacía una posición determinada en la división social del trabajo. Por otro, tenemos que, dada la posición de la mujer en el hogar y las responsabilidades que debe cumplir frente al marido y a los hijos, la mujer que ingresa a la fuerza de trabajo debe así cumplir con una doble jornada: la del trabajo y la del hogar. El dilema, es prepararse para cumplir solamente la tarea del hogar, prepararse en aquellas áreas donde su papel de mujer y su papel de trabajadora impliquen las menores contradicciones o finalmente ubicarse frente al proceso de capacitación profesional en igualdad aparente de condiciones frente al hombre, agregando a esto las otras responsabilidades que debido a su condición de mujer le han sido asignadas en el seno de una sociedad capitalista como la colombiana.

Así, la participación femenina en la educación media es un punto nudal en donde, además de su condición de clase, entra a jugar un papel importante su condición de mujer y su responsabilidad en el proceso de reproducción de la fuerza de trabajo, o sea, responsabilidad frente al marido y a los hijos. Este último aspecto lo analizaremos más adelante.

Analizando los datos correspondientes a la participación de la mujer en el sistema educativo, entre 1935 y 1968, es posible observar en la tabla 3 que si bien en 1935, de 45.670 matriculados 26.726 (58.5\%) fueron hombres y 18.994 (41.5\%) mujeres, en 1968 un $50.5 \%$ de la matrícula total correspondió a los hombres y $49.5 \%$ a las mujeres.

\footnotetext{
${ }^{15}$ DANE, Boletín mensual de estadística, 1975. No. 293, p. 107.
} 


\section{TABLA III \\ EVOLUCION DE LA MATRICULA EN LA EDUCACION MEDIA $1935-1968$}

\begin{tabular}{|r|r|c|c|c|c|}
\hline Años & $\begin{array}{c}\text { Total } \\
\text { Matrícula }\end{array}$ & Hombres & $\%$ & Mujeres & $\%$ \\
\hline 1935 & 45.670 & 26.726 & 58.5 & 18.994 & 41.5 \\
\hline 1945 & 67.877 & 39.651 & 58.4 & 28.226 & 41.6 \\
\hline 1955 & 131.598 & 72.883 & 55.4 & 58.715 & 44.6 \\
\hline 1960 & 243.226 & 131.531 & 54.0 & 111.695 & 46.0 \\
\hline 1965 & 420.130 & 220.109 & 52.4 & 200.021 & 47.6 \\
\hline 1968 & 586.704 & 296.529 & 50.5 & 290.175 & 49.5 \\
\hline
\end{tabular}

Fuente: Cuadro elaborado en base a datos del DANE. Boletín Mensual de Estadística No. 249, abril 1972, página 182.

Al descomponer la matrícula femenina según las modalidades educativas de la educación media (tabla 4), es posible observar que si bien en 1945 solo $19.6 \%$ de la matrícula total de bachillerato correspondió a la mujer, en 1960 las proporciones fueron similares con un $20.4 \%$, pero que en 1975 , la participación de la mujer en la matrícula total de bachillerato había alcanzado un $40 \%$.

TABLA IV

MATRICULA FEMENINA EN EDUCACION MEDIA SEGUN MODALIDAD 1945-1960- 1975

\begin{tabular}{|c|r|r|r|}
\cline { 2 - 4 } \multicolumn{1}{c|}{} & $\mathbf{1 9 4 5}$ & $\mathbf{1 9 6 0}$ & $\mathbf{1 9 7 5}$ \\
\hline Bachillerato & 19.6 & 20.4 & 40.0 \\
\hline Comercial & 58.5 & 68.0 & 67.9 \\
\hline Normalista & 65.9 & 68.2 & 75.4 \\
\hline Industrial & 42.8 & 18.5 & 16.1 \\
\hline Agropecuaria & 0.0 & 7.6 & 17.2 \\
\hline
\end{tabular}

Fuente: Matrícula: Secundaria: Matrícula Oficial Sexo Femenino 1945-1968 DANE. Anuario de Estadísticas Culturales, 1969-1972 Proyecciones DAN E: 1973-75 Secretarías Departamentales.

Gastos: Secundaria: Contraloría General, Informes Financieros Anexos 1945-75: DANE. Estadísticas Fiscales y Administrativas 1945-1968; 1969-75 Proyecciones siguiendo tendencia y con base en el gasto total de Educación Departamental.

Analizando la tabla 4 es posible observar cómo la mayor participación femenina durante el período 1945-1975 se ha dado en la educación normalista, la cual incluso se ha incrementado durante el periodo. En segundo lugar, la mayor participación femenina en relación con la matricula se presenta en' la educación comercial.

En relación con la educación industrial, la mujer ha descendido su participación del $42.8 \%$ en 1945 a $16.1 \%$ en 1975 . Se observa en relación con la educación agropecuaria que ésta pasó de $0 \%$ en 1945 a $17.2 \%$ en 1975. 
Analizando éste período de 30 años, es posible concluir que la tendencia de la participación de la mujer en la educación medía, sí bien ha tenido un cambio significativo en el bachillerato, ha conservado patrones similares en relación con la educación comercial y la normalista.

Sin embargo, los datos muestran que frente al hombre, la participación de la mujer no es tan discriminatoria y que por el contrario, si se tienen en cuenta los datos de matrícula total en este nivel, ya en 1968 ésta era bastante equilibrada entre los sexos.

La discriminación fundamental de la educación media no se ubica, como hemos visto, en relación a los que participan en ella, sino fundamentalmente en relación a los hombres y mujeres que no tienen acceso a la educación. En efecto, el porcentaje total de la población entre los 12 y los 19 años, que en 1951 participó en la educación media, fue solamente de $5.79 \%$. De ese sector de población matriculada, el $5.65 \%$ correspondió a la población total masculina y $5.93 \%$ a la femenina. En 1964, solo participé un total de $13.7 \%$ con un $13.73 \%$ correspondiente a la población masculina y un $13.68 \%$ a la femenina. En 1973 , solamente un $25.36 \%$ de la población de este cohorte de edad asistía a la escuela y representaba el $25.36 \%$ de los hombres y el $24.68 \%$ de las mujeres entre los 12 y los 19 años $^{16}$.

\section{Nivel de universidad}

A nivel universitario la participación de la mujer, como ya se indicó antes, está estrechamente relacionada con el origen de clase de su familia. En relación con ello, Melo ${ }^{17}$ encontró que la mujer logra una mayor participación en aquellas universidades que sirven a las clases más altas ${ }^{18}$. Según ella, la Universidad de los Andes, la del Rosario, la Javeriana y la Bolivariana, matricularon en conjunto el $34.8 \%$ de la población femenina universitaria entre 1957 y 1973.

En la Universidad Javeriana según la misma fuente, 4 de cada 10 estudiantes fueron mujeres, mientras que en la Universidad Nacional estas representaron solamente un $19.5 \%$. Esta participación según el origen familiar, parece estar en relación con las tan conocidas estadísticas de que de cada 100 niños de edad escolar solo 1 logra terminar la universidad.

Según el ICFES ${ }^{19}$ las tasas de escolaridad en la educación universitaria han sido de $3.7 \%$ en $1970,4.2 \%$ en $1971,4.8 \%$ en $1972,5.2 \%$ en 1973 y $5.8 \%$ en 1975 . En relación a la participación femenina en la educación universitaria, es posible señalar que ésta ha crecido de manera significativa pasando de un $7.1 \%$ de la matrícula total en 1945 a $16.2 \%$ en 1960 y a $31 \%$ en 1975 .

A nivel universitario la participación femenina se ubica, como en el nivel anterior, básicamente en carreras intermedias de tipo residual. Al hacer un análisis de los egresados universitarios entre 1920 y 1963, por sexos y especialidades, Ochoa encontró

\footnotetext{
${ }^{16}$ DANE, Op. cit., p. 111.

${ }^{17}$ Melo, Stella. Participación de la mujer en el proceso de Educación Superior en Colombia. Bogotá, 1974. Universidad Javeriana, Departamento de Sociología.

${ }_{18}$ Rama (Op. cit., p. 209)señala que el 48.4\% (1972:209)46.4\% de los estudiantes de la universidad de Los Andes eran hijos de profesionales universitarios independientes, empleados con calificación técnica o universitaria, jefes de nivel medio o alto, los cuales representan solo $4 \%$ de la población masculina económicamente activa.

${ }_{19}$ ICFES, La educación en cifras 1970-1974; 1975.
} 
que $73 \%$ de los egresados en Bellas Artes fueron mujeres y que a éstas correspondió también el $58.6 \%$ en Ciencias de la Educación, el $35.7 \%$ en Humanidades, el $34.6 \%$ en Ciencias Exactas y Naturales, el $21.2 \%$ en Ciencias Sociales, el $19.5 \%$ en Medicina y afines, el $7.7 \%$ en Ingeniería y afines, el $5.4 \%$ en Derecho y el $1.8 \%$ en Agricultura y afines.

Esta visión general de la participación femenina en la matrícula educativa muestra claramente que en estos términos no existe una discriminación hacia la mujer en la educación. En efecto, pudo observarse un equilibrio de la matrícula cuando ésta se analizó por sexos.

La discriminación educativa, como fue oportunamente señalado, adquiere una dimensión significativa cuando se compara la población en edad escolar que tiene acceso al sistema, con la población que se quedó fuera de él.

Es obvio, y se demostró a nivel secundario y universitario, que la participación femenina se diferencia de la masculina, no en relación con el volumen de la matrícula sino en su orientación dentro del sistema educativo. El por qué de esta orientación diferencial, no puede ni plantearse ni solucionarse desde dentro del sistema educativo. A fin de cuentas, la tarea del sistema educativo responde a la necesidad de formación de una fuerza de trabajo adecuada a una división social del trabajo cuya naturaleza no depende del sistema educativo, sino del desarrollo mismo de las fuerzas productivas y de las relaciones sociales de las clases, según éstas se estructuren a partir del proceso de producción.

Para plantear el problema de la mujer frente al sistema educativo, es necesario ubicar su posición ante el proceso de producción, posición que no es homogénea para todas las mujeres y, que depende de su posición de clase como lo veremos en el siguiente punto.

\section{La mujer en el proceso de producción formal}

La organización de una sociedad, y en nuestro caso, de una sociedad capitalista, no está solamente orientada a lograr una producción presente sino también a lograr la reproducción de dicha sociedad dentro de los parámetros en que ésta se encuentra organizada. Es decir, en el caso de nuestra sociedad capitalista, de reproducir las relaciones de clase. Así, los individuos deben cumplir diversas tareas tanto en el proceso de producción como en el proceso de reproducción de la sociedad, tareas que han sido distribuidas según la pertenencia de clase de los individuos.

En esta distribución de tareas, la mujer, por su misma naturaleza, ha sido asignada fundamentalmente a las tareas de reproducción de la sociedad, de reproducción de la fuerza de trabajo y confinada básicamente a las tareas del hogar como madre y esposa.

En una sociedad capitalista como la colombiana, que gira en torno a la generación y acumulación de ganancias, el trabajo de la mujer que no genera ganancia carece también de un valor económico realizable en el mercado. El trabajo que la mujer realiza cada día para hacer posible que el esposo se presente en el sitio de trabajo y para hacer posible la socialización de los hijos que participarán en la producción del futuro, no tiene un valor. En el salario de su marido solo se paga una parte del tiempo que él trabaja, y nunca se estipula el valor del tiempo de trabajo que la mujer invirtió en el hogar, para hacer posible el trabajo del marido. 
Esta posición de la mujer en el hogar, en la reproducción de la fuerza de trabajo, es determinante de su ubicación frente a la educación. Por encima de todo, y mientras la sociedad no comparta de manera significativa la responsabilidad de la mujer en la reproducción de la fuerza de trabajo, la educación femenina tenderá a ubicarse en aquellos campos profesionales más acordes con la definición que la sociedad ha hecho de ella, y que le facilitarán el cumplimiento de su responsabilidad fundamental en el hogar.

Las condiciones económicas de la mujer de la clase alta le permite evadir la responsabilidad en el hogar, y en gran parte, su tarea en la reproducción de la fuerza de trabajo y redefinir su posición frente al proceso directo de producción. Es esta redefinición la que ubica nuevas perspectivas educacionales para la mujer de esta clase ${ }^{20}$.

Para aquellas mujeres pertenecientes a otras clases sociales, su participación en el proceso directo de producción no implica una liberación de su posición en el proceso de reproducción. De ahí que se vea abocada a cumplir una doble responsabilidad: frente al hogar y frente al sitio de trabajo, debe cumplir una doble jornada ${ }^{21}$. Más aún, el hecho de que no haya roto con sus responsabilidades en el hogar, cuyo trabajo como ya vimos carece de valor económico, lo afecta en este proceso, y generalmente recibe por un trabajo similar un salario diferente al del hombre.

Concluyendo el análisis podemos señalar:

1. Que para ubicar correctamente los pasos y las tareas de la mujer en relación a su posición en la sociedad, es necesario partir de una ubicación, lo más correcta posible, de las causas de esta posición de discriminación.

2. Que la posición discriminatoria de la mujer en la sociedad no afecta de la misma manera a todas las mujeres, sino que según la posición de clase de la mujer, la discriminación se materializa de manera diferente.

3. Que es casi imposible redefinir la posición de la mujer en la sociedad sin redefinir su posición y su responsabilidad en la reproducción de ésta.

4. Que los esfuerzos promovidos para lograr una participación más amplia de la mujer en el sistema educativo, afectarán de manera diferente al sector femenino según su posición de clase.

\section{Epilogo}

En la proyección de políticas de acción para la mujer; en relación con el campo de la educación se presentan básicamente dos alternativas que parten de los diferentes enfoques sobre la posición de la mujer en el sistema educativo que fueron señalados anteriormente.

\footnotetext{
${ }^{20}$ En "la clase alta" el ingreso estimado por trabajo doméstico apenas representa una quinta parte de la renta familiar, en tanto que en las "clases bajas" vale más el trabajo doméstico, así sea estimado a niveles mínimos de salario, que el trabajo en la producción mercantil. Buenaventura, Nicolás. 1975. "La mujer obrera en el movimiento democrático femenino" En Estudios Marxistas. No. 10, pp: 47-63.

${ }^{21}$ Según la información recogida por las obreras de FEDETEX en Medellín, solamente $3 \%$ utiliza servicio doméstico para la limpieza, $3 \%$ para ayuda en la cocina; ninguna para los niños y $14 \%$ para el arreglo de la ropa. (Buenaventura, 1976:54).
} 
Por un lado, abogar por una igualdad de oportunidades frente al hombre en todas las especialidades educativas. Esta posición conduciría a que se demanden políticas educativas homogéneas para satisfacer necesidades de diferente naturaleza, urgencia y dimensión, bajo la presunción de que la obtención de igualdad dentro del sistema educativo puede llegar a eliminar las desigualdades que se originan a nivel del sistema total. Esta posición, que ubica la solución dentro del sistema educativo, en el fondo lo que hace es mantener y reforzar la existencia de clases sociales a las cuales, en el mejor de los casos, el sistema educativo estaría en condiciones de satisfacer "sus" necesidades, pero las que en realidad no vienen a ser las de los grupos en sí, sino necesidades definidas a partir de las relaciones de producción dominantes.

Por otro lado, teniendo en cuenta las relaciones de clase, la segunda alternativa se coloca por fuera del sistema educativo, ubicando la lucha contra la discriminación que se da en éste, en el terreno de la igualdad de participación en los niveles económico, político y social de la sociedad. Lo anterior incidiría en la igualdad dentro del sistema educativo y no lo contrario. Dentro de este contexto, se visualizaría una real participación en el proceso educativo no tradicional, en él surge la actividad directa de las mujeres de todos los sectores de clase y en pie de igualdad en los procesos políticos (más allá del voto), en los procesos económicos (fundamentalmente en los procesos de toma de decisiones) y a nivel social, en igualdad de derechos y de obligaciones junto con el hombre.

Podría aducirse que para llegar a lo anterior, se necesita la educación formal. Sin embargo, quien haya observado la participación de la mujer en algunos de los procesos atrás enunciados, específicamente a nivel de base, podrá darse cuenta que, el no tener una educación institucionalizada, no ha sido limitante y en cambio sí se considera que esa participación activa y directa, en dichos procesos, constituye en sí el mejor y más adecuado pro-cesa educativo. Esto, por cuanto no conlleva ni las limitaciones ni las tendencias del proceso educativo tradicional, marcadamente alienante para la mujer y para el hombre de las clases dominadas. 